\title{
Freshwater biodiversity in western Nepal: A review
}

\author{
Janak Raj Khatiwada ${ }^{1}$ (D) Jagan Nath Adhikari ${ }^{2,3 *} \mathbb{E} \mid$ Deepak Rijal ${ }^{4} \mid$ Lila Nath Sharma $^{5}(\mathbb{D}$
}

\author{
${ }^{1}$ Department of Biology, Lakehead University, 955 Oliver Road, Thunder Bay, ON, P7B 5E1, Canada \\ ${ }^{2}$ Central Department of Zoology, Institute of Science and Technology, Tribhuvan University, Kathmandu, Nepal \\ ${ }^{3}$ Birendra Multiple Campus, Bharatpur, Chitwan, Nepal \\ ${ }^{4}$ USAID Program for Aquatic Natural Resource Improvement, Paani Program, Baluwatar, Kathmandu, Nepal \\ ${ }^{5}$ ForestAction Nepal, Lalitpur, Nepal \\ *Correspondence: jagan.adhikari@bimc.tu.edu.np
}

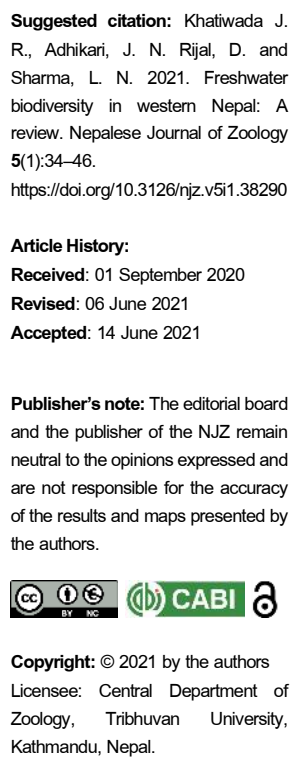

wetlands regulate the water cycle and have a unique role in maintaining the food webs (Mitsch et al. 2015). With this realization the Convention on Biological Diversity 1992 has given a high priority to wetlands conservation as the services they provide directly contribute to the livelihoods of the people (Bell 1972).

Globally, over 126,000 described species of fish, amphibians, reptiles, birds, mammals, molluscs, insects, and plants inhabit freshwater systems, and they are concentrated in less than $1 \%$ of the world's surface area (Baillie et al. 2008). Despite their immense significance, freshwater ecosystems are among the most vulnerable ecosystems on the planet (Geist 2011). These systems face serious threats due to increased human population, economic growth and global environmental changes (Vörösmarty et al. 2010). Major anthropogenic stressors in freshwater ecosystem include the construction of dams, pollution, invasive species, and overharvesting (Junk et al. 2013). These threats on freshwater ecosystems not only diminish the quality and quantity of services including food security and nutrition, which are essential to human populations (Lamsal et al. 2015) but also affect their biodiversity (Allen et al. 2010). Declines in biodiversity are far greater in freshwater than terrestrial ecosystems and marine realms (Vörösmarty et al. 2010).

Nepal, with its rich water resources distributed over the landscape, has contributed to create ecologically valued wetlands. The country's freshwater habitats cover about 745,000 hectares ( $5 \%$ of the total area) that comprises rivers, lakes, ponds, wetlands, reservoirs, and irrigated rice fields (Sharma 2008). The total drainage area by rivers of Nepal is estimated to be about $194,471 \mathrm{~km}^{2} ; 76 \%$ of the area lies within Nepal (WECS 2003; DoFD 2011; ICIMOD 2014) (Table 1). About $74 \%$ of the area in the country is drained by the four major rivers, viz., Koshi, Gandaki, Karnali, and Mahakali (Fig. 1). Among them, Karnali River provides the largest drainage area $(28.46 \%)$ in comparison to other rivers in Nepal.

Freshwater ecosystems provide a wide range of habitats and harbor a large proportion of plants and animals (Dudgeon et al. 2006; Collen et al. 2014). Both wetlands and terrestrial habitat of western highlands support 56 species of mammals, 288 species of birds, 11 species of herpetofauna, and three species of fish; likewise, western mid-hills support 55 mammals, 388 birds, 80 herpetofauna and 17 fish, similarly western Terai and Siwalik support 64 mammals, 469 birds, 48 herpetofauna and 77 fish 


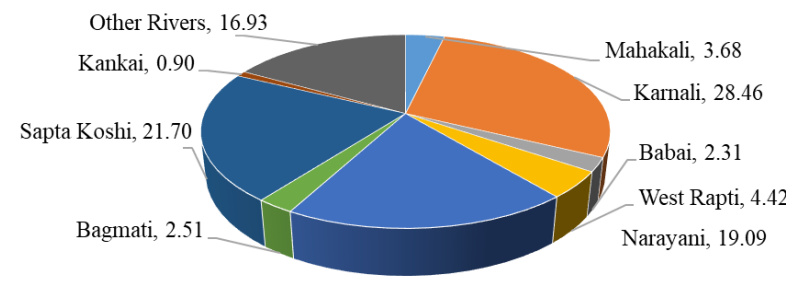

Figure 1. Drainage area (\%) covered by the river in Nepal (Source: DoFD 2011).

(Bhuju et al. 2007; Kunwar et al. 2016). However, due to a lack of proper assessment of the distribution of freshwater diversity, formulation of conservation and management strategies are constrained in western Nepal. Conservation plans and programs have given due attention mainly to terrestrial biodiversity. There are seven Protected Areas - Five National Parks (Banke, Bardia, Suklaphanta, Rara and Shey Phoksundo) and two Conservation Areas (Api-Nampa and Krishnasar Conservation Area) in western Nepal. The Department of National Parks and Wildlife Conservation (DNPWC), Ministry of Forest and Environment, Government of Nepal prepares the action and management plans for the management of National Parks and Conservation Areas (DNPWC 2020a). The majority of these action plans and management plans focus on terrestrial ecosystems, large mammals, and birds, while freshwater biotic communities hardly draw the attention of the planners. To effectively implement conservation programs for freshwater biodiversity, it is important to understand the value and distribution of diversity across water bodies, including the identification of species and their distribution inside and outside of the protected areas (Rodrigues et al. 2004). The freshwater of Karnali and Mahakali river systems have been utilized for economic purposes such as irrigation, hydroelectricity, and river-based ecotourism, which at the same time are critical habitats for freshwater biodiversity. The Karnali and Mahakali river basins are the most biologically diverse ecosystems for freshwater fish, amphibians, reptiles, birds, and mammals with high endemism (Dubois 1973; Dubois \& Matsui 1983; Terashima 1984). This rich biodiversity could be explained by its large altitudinal gradients $(100 \mathrm{~m}$ - above $7132 \mathrm{~m}$ ) linked to the

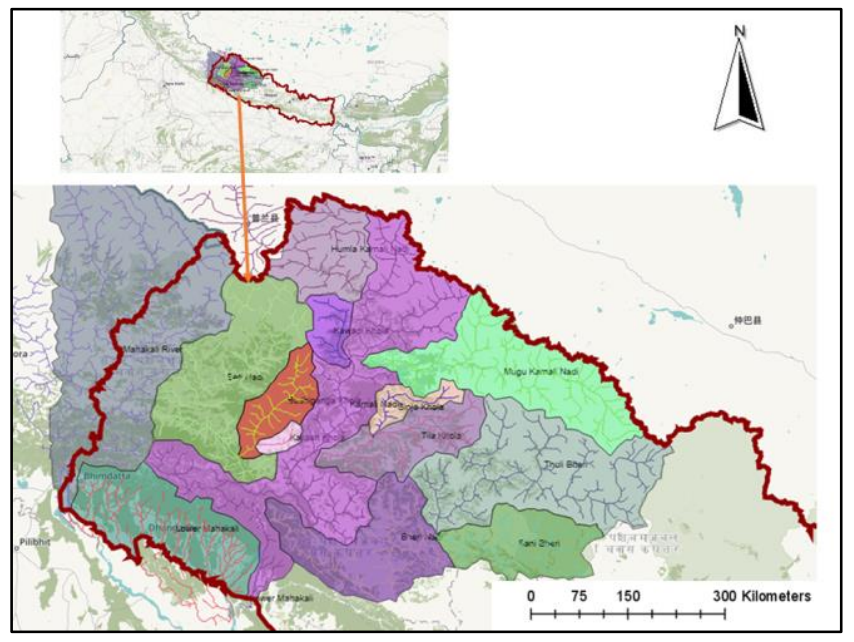

Figure 2. Map of Karnali and Mahakali river basins (Source: ICIMOD 2014) creation of a variety of climatic conditions (Dobremez 1976; Grytnes 2003). Also, there are unique habitats throughout the region, such as productive flooded grassland and forests, oxbow lakes, clear and white-water streams, which promote higher biological diversity and endemism. In the Himalayan region, a large proportion of the landscape has been transformed over a long period of time by human activities such as cultivation, grazing, and extraction of natural resources (Ives 2004; Byers 2005). These human-induced activities threaten its long-term viability, the integrity of its biodiversity, and the ecosystem services that human populations have been deriving.

Studies elsewhere have shown that certain freshwater species could be used as proxy/indicators of habitat quality (Abell et al. 2011; Bogardi et al. 2020); however, the research on this aspect in Nepal is scanty. Although increasing efforts to estimate the richness of freshwater vertebrate species at a global scale has been witnessed (Collen et al. 2014), the comprehensive information on overall aquatic biodiversity crucial for the management and conservation interventions at smaller geographical scales (i.e., watershed, streams, rivulets) is limited in Nepal and especially in the Karnali and Mahakali river basins is largely missing (Fig. 2). These river basins offer a striking example of the ecological and socio-economic complexities that challenge conservation of lotic ecosystems in western Nepal. However, a paucity of systematically documented information about freshwater species and their distribution could hinder the development and implementation of conservation and management plans for Western Nepal. This review discusses i) the distribution and physical characteristics of freshwater ecosystems (lakes and rivers) of western Nepal, ii) ecosystem services and biodiversity of wetlands of western Nepal, iii) threats to freshwater ecosystems and conservation history of wetlands exploration.

\section{2 | Extent and distribution of freshwater ecosystem}

\section{in Western Nepal}

In Nepal, rivers occupy the largest proportion based on the total area covered (53\%) followed by the irrigated paddy fields (Fig. 3). Available literature suggests that there are more than 6000 rivers, 3252 glaciers, 2323 glacial lakes, 5358 tectonic and ox-

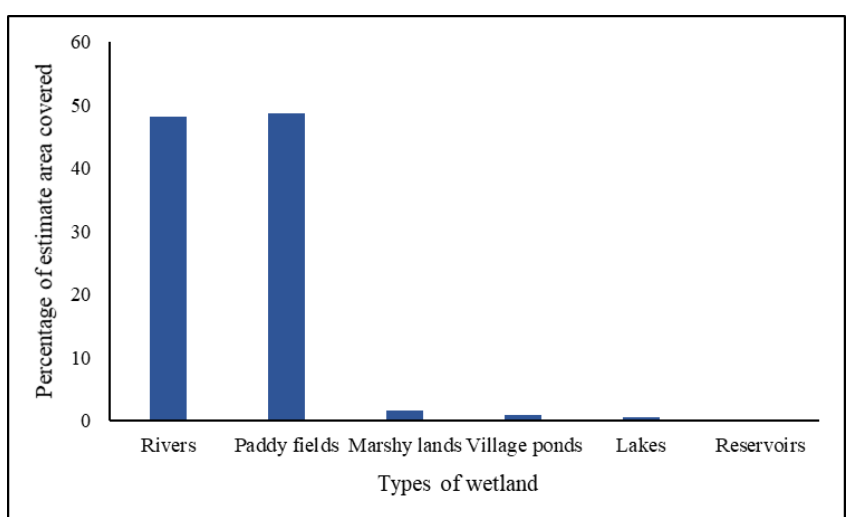

Figure 3. Estimated area covered by wetlands in Nepal (Source: DoFD 2011) 
Table 1. Estimated runoff from the rivers of Nepal (ICIMOD 2014)

\begin{tabular}{|c|c|c|c|c|c|c|}
\hline \multirow{2}{*}{$\begin{array}{l}\text { S. } \\
\text { N. }\end{array}$} & \multirow{2}{*}{ River } & \multirow{2}{*}{$\begin{array}{l}\text { Length } \\
(\mathrm{km})\end{array}$} & \multicolumn{2}{|c|}{ Drainage area $\left(\mathrm{km}^{2}\right)$} & \multicolumn{2}{|c|}{$\begin{array}{l}\text { Estimated Runoff } \\
\left(\mathrm{m}^{3} / \mathrm{sec}\right)\end{array}$} \\
\hline & & & Total & Nepal & $\begin{array}{l}\text { From all } \\
\text { Basins }\end{array}$ & $\begin{array}{l}\text { From } \\
\text { Nepal }\end{array}$ \\
\hline 1 & Mahakali & 223 & 15,260 & 5,410 & 698 & 247 \\
\hline 2 & Karnali & 507 & 44,000 & 41,890 & 1441 & 1371 \\
\hline 3 & Babai & 190 & 3,400 & 3400 & 103 & 103 \\
\hline 4 & West Rapti & 257 & 6,500 & 6,500 & 224 & 224 \\
\hline 5 & Narayani & 332 & 34,960 & 28090 & 1753 & 1409 \\
\hline 6 & Bagmati & 163 & 3,700 & 3,700 & 178 & 178 \\
\hline 7 & Sapta Koshi & 513 & 60,400 & 31940 & 1658 & 878 \\
\hline 8 & Kankai & 108 & 1330 & 1330 & 68 & 68 \\
\hline 9 & Other rivers & & 24921 & 24921 & 1001 & 1001 \\
\hline \multicolumn{3}{|c|}{ Total } & 194,471 & 147,181 & 7125 & 5479 \\
\hline
\end{tabular}

bow lakes in Nepal (NLCDC 2019). Among them, 1271 lakes $(23.72 \%)$ were recorded from the Sudurpaschim and Karnali provinces of western Nepal (Bhuju et al. 2010). The map-based inventory showed that 75 districts out of 77 districts contain lakes. Sixteen districts have over 100 lakes in each (NLCDC 2020).

More than 6,000 rivers, streams, rivulets, brooks, and channels (nalas) of Nepal have a linear length of approximately 45,000 km. Among them, $66 \%$ of the total rivers have been originated from Nepal while others have been originated from the Tibetan Plateau. The total drainage area occupied by rivers and watersheds is $191,000 \mathrm{~km}^{2}, 70 \%$ of which lies in Nepal. Nepal has 24 rivers which have more than $500 \mathrm{~km}$ in length. Likewise, more than 100 rivers are approximately $160 \mathrm{~km}$ long, and more than 1000 rivers are $11 \mathrm{~km}$ long on average (Table 1)

Nepal's permanent lakes are broadly categorized into the glacial, tectonic and ox-bow lakes (IUCN Nepal 2004; Lacoul \& Freedman 2005). Glacial lakes formed from the glacial activities are located in the high mountain regions. Tectonic lakes were formed due to tectonic activities of the earth and are largely distributed across mid-hills, whereas ox-bow lakes are found mostly in the lowland areas of the southern plain in the Terai region (Lacoul \& Freedman 2005). These ox-bow lakes are mainly formed due to the impoundment of rivers (IUCN Nepal 2004). Overall, the surface area and the number of glacial lakes is the lowest (total 241) in the Gandaki river basin compared to the Koshi, and Karnali river basins (Khadka et al. 2018).

\section{1 | Lakes of western Nepal}

The Karnali river basin has 645 glacial lakes that cover around $33.50 \mathrm{~km}^{2}$, and the Mahakali river basin has 18 glacial lakes that cover around $0.33 \mathrm{~km}^{2}$ surface area (Khadka et al. 2018) (Table 2). Highlands of Sudhurpaschim and Karnali province have 917 lakes $(74.49 \%)$ followed by mid-hills $115(9.34 \%)$ and Terai and Siwalik 199 (16.16\%). Humla district has the highest number of lakes ( $n=381)$ while Kalikot $(n=1)$ and Baitadi $(n=1)$ have the least number of lakes (Bhuju et al. 2010) (Table 2).
Table 2. Number of lakes recorded as ecological zones and districts from Sudurpaschim and Karnali Province of Western Nepal (NLCDC 2020).

\begin{tabular}{|c|c|c|c|c|c|}
\hline Area & $\begin{array}{l}\text { Ecologica } \\
\text { I zones }\end{array}$ & District & $\begin{array}{l}\text { Total } \\
\text { lakes }\end{array}$ & Famous lakes & Remarks \\
\hline \multirow{30}{*}{ 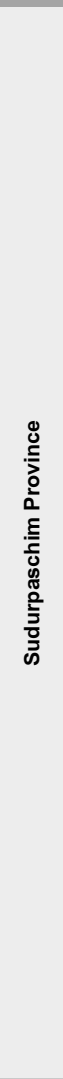 } & \multirow{2}{*}{ Highland } & \multirow{2}{*}{ Bajhang } & \multirow{2}{*}{25} & Surma Sarovar & Holy lake \\
\hline & & & & Tima Daha & \\
\hline & \multirow{5}{*}{ Highland } & \multirow{5}{*}{ Bajura } & \multirow{5}{*}{57} & Chhede Daha & \\
\hline & & & & Buddhinanda & \\
\hline & & & & Kailash Taal & Holy lake \\
\hline & & & & Rakshyas & \\
\hline & & & & Masta Daha & \\
\hline & \multirow{2}{*}{ Highland } & \multirow{2}{*}{ Darchula } & \multirow{2}{*}{19} & Brahama Daha & Holy lake \\
\hline & & & & Pasa Daha & \\
\hline & \multirow{5}{*}{ Midhill } & \multirow{5}{*}{ Achham } & \multirow{5}{*}{13} & Khaptad & Holy lake \\
\hline & & & & Ramaroshan & Holy lake \\
\hline & & & & Kalidaha & \\
\hline & & & & Batulee & \\
\hline & & & & Barha Banda & Holy lake \\
\hline & \multirow{3}{*}{ Midhill } & \multirow{3}{*}{ Dadeldhura } & \multirow{3}{*}{3} & $\begin{array}{l}\text { Ali Taal } \\
\text { (Dadeldhura) }\end{array}$ & Holy lake \\
\hline & & & & Bodapanthi Taal & \\
\hline & & & & Nayal Tal & \\
\hline & Midhill & Baitadi & 1 & Baite Tal & \\
\hline & \multirow{3}{*}{ Midhill } & & & Khaptad Lake & Holy lake \\
\hline & & Doti & 19 & Ali Taal (Doti) & Holy lake \\
\hline & & & & Chatiwan Lake & \\
\hline & & & & Jokhar Lake & \\
\hline & $\begin{array}{l}\text { Terai- } \\
\text { Siwalik }\end{array}$ & Kailali & 114 & $\begin{array}{l}\text { Ghodaghodi } \\
\text { Lake }\end{array}$ & $\begin{array}{l}\text { Ramsar } \\
\text { Site }\end{array}$ \\
\hline & & & & $\begin{array}{l}\text { Muchheli River } \\
\text { (Godawariseti) }\end{array}$ & \\
\hline & & & & Jhilmila Taal & Holy lake \\
\hline & & & & Bedkot Taal & Holy lake \\
\hline & Terai- & & & Mudka Taal & \\
\hline & Siwalik & Kanchenpur & 85 & Rani Taal & \\
\hline & & & & Sundeu Taal & \\
\hline & & & & Kamalpokhari & \\
\hline & & & & Phoksundo Lake & Ramsar site \\
\hline & & & & Pritha Daha & \\
\hline & Highland & Dolpa & 210 & Jagadulla & \\
\hline & & & & Matae Taal & \\
\hline & & & & Chhamkuni Taal & \\
\hline & Highland & Humla & 381 & Selima Chho & \\
\hline & & & & Jumla Lake & \\
\hline & & & & Giridaha & \\
\hline & Hiabland & lumla & 99 & Thakurjyu Daha & \\
\hline & Highland & Jumla & 99 & Sanka Daha & \\
\hline & & & & Pani Pokhari & \\
\hline & & & & Bistajyu Daha & \\
\hline 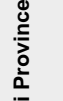 & Highland & Mugu & 125 & Rara Lake & $\begin{array}{l}\text { Largest } \\
\text { lake, } \\
\text { Ramsar site }\end{array}$ \\
\hline స్ & & & & Dudh Taal & \\
\hline 苙 & & & & Kunya Lake & \\
\hline & Midhill & Dailekh & 7 & Khal Taal & \\
\hline & IVIIanilI & Lallekn & $r$ & Bada Pokhari & \\
\hline & & & & Tal Pokhari & \\
\hline & Midhill & Salvan & 5 & Kubinde Daha & Holy lake \\
\hline & & & & Kachhuwa Daha & \\
\hline & Midhill & Surkhet & 22 & Bulbule Lake & \\
\hline & & & & Syarpu Taal & Holy lake \\
\hline & Midhill & Western & 70 & Kamal Taal & \\
\hline & IVIIanilli & Rukum & 10 & Chhipri Daha & \\
\hline & & & & Thuli Daha & \\
\hline & Midhill & Jajarkot & 16 & Kali Raha & \\
\hline & Highland & Kalikot & 1 & Pan Daha & \\
\hline
\end{tabular}

Many lakes in western Nepal (Sudurpaschim and Karnali provinces), such as Ramaroshan lake complex, Gididaha, Jhilmila, Bedkot, SatiKarnali etc. have cultural and biological significant values (Chalaune et al. 2020). The largest (Rara Lake) and the beautiful Shey- 


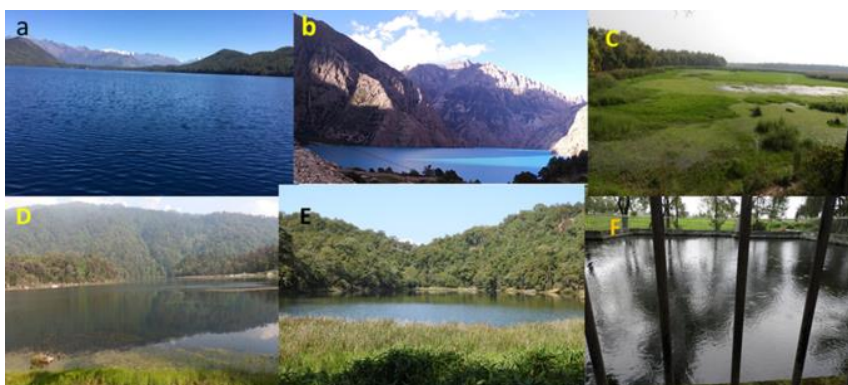

Figure 4. Some famous lakes of Western Nepal: a- Rara Lake (the largest lake of Nepal and Ramsar site, Photo credit: K. P. Kandel), b- Phoksundo Lake (the Ramsar site, Photo credit: K. C. Ghimire), c- Rani Lake (largest lake of Sukla Phanta National Park, Photo credit: J. N. Adhikari), d- Ramaroshan Lake complex (Jingale Lake, Photo credit: J. N. Adhikari), e- Jhilmila Lake (The holy lake of Kanchanpur, Photo credit: J. N. Adhikari), f- Bulbule Lake (Famous lake of Surkhet, Photo credit: J. N. Adhikari).

Phoksundo Lake of Nepal also lie in the western region which are also listed as Ramsar sites (MoFE 2018; NLCDC 2020) (Fig. 4).

\section{2 | Rivers of western Nepal}

The western region of Nepal is drained by two major river basins Karnali $(550 \mathrm{~km}$ ) and Mahakali $(223 \mathrm{~km}$ ) (ICIMOD 2014, Table 3). The Karnali River is also called Saptakarnali or Geruwa in Nepal and Ghaghara in India, formed by seven major tributaries and other associated rivers; Bheri and Seti are the major tributaries. The Karnali drains western Nepal, andthe upper Bheri river drains Dolpo valley. Likewise, Mahakali or Kali River is the union of major rivers named Surma, Chamelia, and Lipu river and runs along the Nepal-India border on the west and joins Karnali in India, where this river is called Ghaghara (ICIMOD 2014) (Fig. 2).

\section{3 | Ecosystem services}

Carbon and nutrient cycling in streams and lakes are largely controlled by aquatic invertebrate consumers that largely feed on organic matter, graze on periphyton, or consume dead organic matter (Atkinson et al. 2017). Therefore, the ecosystem's functioning is highly dependent on human dimensions and other environmental characteristics. This review discusses the major goods and services provided by freshwater resources.

\section{1 | Water services}

Wetlands provide an incessant flow of ecosystem services and directly support millions of people to sustain their lives (Dudgeon et al. 2006; Sharma et al. 2015; Olalde et al. 2019). In Nepal, a total of 22 wetlands dependent communities such as Bantar (Sardar), Barhamans, Bote, Danuwar, Darae, Gongi, Jhagat, Kewat, Khanwas (Rhanjhi), Khuna, Kumal, Kusahar (Kusaha), Manjhi, Dusadh, Mallaha, Mukhiya (Bihin), Musahar, Pode, Sahani, Satar, Sunaha, and Tharu depend on lakes and wetlands for their survival (NLCDC 2020). Ecosystem goods (provisioning services) provided by the aquatic ecosystem/wetlands are mainly: water for drinking, irrigation, generation of hydroelectricity, fisheries, non-timber forest products, and recreation. Other services include flood control, groundwater recharge, carbon sequestration, nutrient removal, and biodiversity maintenance (Naiman \& Turner 2000).

\section{Hydroelectricity}

In Nepal, the perennial nature of rivers estimated up to 170 billion $\mathrm{m}^{3}$ annual runoff flows from steep gradients and rugged topography. The estimated feasible hydropower potential is estimated 45610 Mega Watt (MW), which is equivalent to $50 \%$ of the total theoretical potential (83290 MW) (Bhatt 2017; DoED 2020).

The production of hydroelectricity is low in western Nepal. The government of Nepal purposed the Pancheshowar Multipurpose Project in the Mahakali River. Pancheshowar Multipurpose Project has been identified as a huge storage scheme to be developed to maximize peak power benefit in the order of 6,720 MW (Pancheshowar High Dam- 6480 MW and Rupali Gad ReRegulating Dam- $240 \mathrm{MW}$ ) with an annual average energy production of 12,333-Gigawatt hour (GWh) (DoED 2020). The government of Nepal has proposed to construct more than 46 hydropower plants of more than $1 \mathrm{MW}$ from Western Nepal that can produce 6923.98 MW electricity (DoED 2020) (Table 4).

\section{Irrigation}

Irrigation is an important ecosystem service of the wetland. Nepal has a total of $2,642,000$ ha cultivated land ( $18 \%$ of its land area), of which only two-thirds $(1,766,000 \mathrm{ha})$ of land is potentially irrigable (Poudel \& Sharma 2012). The report of WECS (2011) showed that $42 \%$ of the total cultivated area has irrigation of some sort (only in the rainy season), whereas $17 \%$ of the cultivated area has year-round irrigation.

The famous Rani Jamara Kulariya Irrigation Scheme from Karnali River, located in Kailali District in the Far Western Terai Region of Nepal, provided more efficient, reliable, and flexible water services to farmers and households. About 25,000 farming households comprising close to 160,000 people are benefited from this project. This irrigation scheme irrigates more than 14,300 ha of farmland (The World Bank 2019). Another national pride irrigation project is the Sikta irrigation project which will irrigate about 43,000 ha of land of Banke district. The construction of the $45 \mathrm{~km}$ western canal of the project has been

Table 3. Number and surface area $\left(\mathrm{km}^{2}\right)$ of glacial lakes in Karnali and Mahakal river basins of western Nepal in 2017 (Khadka et al. 2018).

\begin{tabular}{|c|c|c|c|c|}
\hline SN & River basin & Sub basin & Numbers & $\begin{array}{c}\text { Total surface area } \\
\left(\mathbf{k m}^{2}\right)\end{array}$ \\
\hline \multirow[t]{8}{*}{1} & \multirow[t]{7}{*}{ Karnali } & Humla Karnali & 237 & 12.63 \\
\hline & & Mugu Karnali & 174 & 5.65 \\
\hline & & Kawari & 21 & 0.95 \\
\hline & & $\begin{array}{l}\text { Karnali sub } \\
\text { part }\end{array}$ & 4 & 0.3 \\
\hline & & Tila & 57 & 3.76 \\
\hline & & Bheri & 129 & 9.09 \\
\hline & & West Seti & 23 & 1.12 \\
\hline & Total & & 645 & 33.5 \\
\hline 2 & Mahakali & & 18 & 0.33 \\
\hline
\end{tabular}


Table 4. Proposed Hydropower project in Western Nepal (NPC 2020)

\begin{tabular}{|c|c|c|c|c|c|c|c|c|}
\hline S.N. & Project & $\begin{array}{l}\text { Capacity } \\
\text { (MW) }\end{array}$ & River & \multicolumn{2}{|l|}{ Latitude $\mathbf{N}$} & \multicolumn{2}{|l|}{ Longitude E } & District \\
\hline 1 & Middle Budhiganga HEP & 5 & Budhi Ganga & $29^{\circ} 18^{\prime} 36^{\prime \prime}$ & $29^{\circ} 20^{\prime} 29^{\prime \prime}$ & $81^{\circ} 17^{\prime} 30^{\prime \prime}$ & $81^{\circ} 19^{\prime} 15^{\prime \prime}$ & Achham, Bajura \\
\hline 3 & Mugu Karnali Storage HEP & 1902 & Karnali & $29^{\circ} 23^{\prime} 43^{\prime \prime}$ & $29^{\circ} 41^{\prime} 22^{\prime \prime}$ & $81^{\circ} 39^{\prime} 14^{\prime \prime}$ & $81^{\circ} 57^{\prime} 13^{\prime \prime}$ & $\begin{array}{l}\text { Bajura, Humla, } \\
\text { Mugu }\end{array}$ \\
\hline 5 & Seti Nadi-3 HEP & 65 & Seti Khola & $29^{\circ} 30^{\prime} 00^{\prime \prime}$ & $29^{\circ} 36^{\prime} 00^{\prime \prime}$ & $81^{\circ} 07^{\prime} 51^{\prime \prime}$ & $81^{\circ} 15^{\prime} 00^{\prime \prime}$ & Bajhang \\
\hline 6 & Upper Sunigad HEP & 8.42 & Suni Gad & $29^{\circ} 40^{\prime} 05^{\prime \prime}$ & $29^{\circ} 41^{\prime} 35^{\prime \prime}$ & $81^{\circ} 12^{\prime} 15^{\prime \prime}$ & $81^{\circ} 14^{\prime} 00^{\prime \prime}$ & Bajhang \\
\hline 7 & Bajhang Upper Seti HEP & 140 & Seti & $29^{\circ} 44^{\prime} 50^{\prime \prime}$ & $29^{\circ} 50^{\prime} 30^{\prime \prime}$ & $81^{\circ} 15^{\prime} 00^{\prime \prime}$ & $81^{\circ} 18^{\prime} 45^{\prime \prime}$ & Bajhang \\
\hline 8 & Chainpur Seti HEP & 210 & Seti Khola & $29^{\circ} 36^{\prime} 20^{\prime \prime}$ & $29^{\circ} 44^{\prime} 40^{\prime \prime}$ & $81^{\circ} 16^{\prime} 00^{\prime \prime}$ & $81^{\circ} 20^{\prime} 15^{\prime \prime}$ & Bajhang \\
\hline 9 & Humla Karnali 1 HEP & 235 & Humla Karnali & $29^{\circ} 41^{\prime} 30^{\prime \prime}$ & $29^{\circ} 47^{\prime} 00^{\prime \prime}$ & $81^{\circ} 56^{\prime} 30^{\prime \prime}$ & $82^{\circ} 02^{\prime} 00^{\prime \prime}$ & Humla \\
\hline 10 & Humla Karnali 2 HEP & 335 & Humla Karnali & $29^{\circ} 46^{\prime} 00^{\prime \prime}$ & $29^{\circ} 52^{\prime} 47^{\prime \prime}$ & $81^{\circ} 51^{\prime} 00^{\prime \prime}$ & $81^{\circ} 56^{\prime} 29^{\prime \prime}$ & Humla \\
\hline 12 & Chuwa Khola HEP & 70 & Chuwa & $29^{\circ} 56^{\prime} 30^{\prime \prime}$ & $29^{\circ} 57^{\prime} 45^{\prime \prime}$ & $81^{\circ} 51^{\prime} 30^{\prime \prime}$ & $81^{\circ} 56^{\prime} 05^{\prime \prime}$ & Humla \\
\hline 13 & Gidi Khola HEP & 9 & Gidi & $29^{\circ} 11^{\prime} 12^{\prime \prime}$ & $29^{\circ} 13^{\prime} 55^{\prime \prime}$ & $82^{\circ} 09^{\prime} 11^{\prime \prime}$ & $82^{\circ} 10^{\prime} 55^{\prime \prime}$ & Jumla \\
\hline 14 & Upper Gidi Khola HEP & 3.3 & Gidi & $29^{\circ} 09^{\prime} 30^{\prime \prime}$ & $29^{\circ} 10^{\prime} 59^{\prime \prime}$ & $82^{\circ} 08^{\prime} 30^{\prime \prime}$ & $82^{\circ} 10^{\prime} 20^{\prime \prime}$ & Jumla \\
\hline 15 & Mugu Khola HEP & 57 & Mugu & $29^{\circ} 35^{\prime} 27^{\prime \prime}$ & $29^{\circ} 38^{\prime} 45^{\prime \prime}$ & $82^{\circ} 25^{\prime} 55^{\prime \prime}$ & $82^{\circ} 31^{\prime} 15^{\prime \prime}$ & Mugu \\
\hline 16 & Namlan Khola HEP & 260 & Namlan & $29^{\circ} 33^{\prime} 35^{\prime \prime}$ & $29^{\circ} 35^{\prime} 27^{\prime \prime}$ & $82^{\circ} 26^{\prime} 10^{\prime \prime}$ & $82^{\circ} 37^{\prime} 00^{\prime \prime}$ & Mugu \\
\hline 17 & Upper Mugu Karnali HEP & 306 & Mugu Karnali & $29^{\circ} 33^{\prime} 00^{\prime \prime}$ & $29^{\circ} 35^{\prime} 50^{\prime \prime}$ & $82^{\circ} 13^{\prime} 45^{\prime \prime}$ & $82^{\circ} 24^{\prime} 00^{\prime \prime}$ & Mugu \\
\hline 18 & Mugu Karnali HEP & 159.62 & Mugu Karnali & $29^{\circ} 34^{\prime} 37^{\prime \prime}$ & $29^{\circ} 37^{\prime} 39^{\prime \prime}$ & $81^{\circ} 51^{\prime} 22^{\prime \prime}$ & $82^{\circ} 06^{\prime} 46^{\prime \prime}$ & Mugu \\
\hline 19 & Upper Bheri PROR HEP & 281 & Thuli Bheri & $28^{\circ} 58^{\prime} 00^{\prime \prime}$ & $29^{\circ} 02^{\prime} 32^{\prime \prime}$ & $82^{\circ} 35^{\prime} 00^{\prime \prime}$ & $82^{\circ} 52^{\prime} 52^{\prime \prime}$ & Dolpa \\
\hline 20 & Tarap Khola HEP & 50 & Tarap & $28^{\circ} 53^{\prime} 45^{\prime \prime}$ & $28^{\circ} 57^{\prime} 00^{\prime \prime}$ & $83^{\circ} 01^{\prime} 55^{\prime \prime}$ & $83^{\circ} 04^{\prime} 25^{\prime \prime}$ & Dolpa \\
\hline 21 & Jagdulla HEP & 106 & Bheri & $29^{\circ} 03^{\prime} 16^{\prime \prime}$ & $29^{\circ} 07^{\prime} 53^{\prime \prime}$ & 820 33' 43" & $82^{\circ} 38^{\prime} 00^{\prime \prime}$ & Dolpa, Jajarkot \\
\hline 22 & Jagdulla A HEP & 82.3 & Bheri & $28^{\circ} 59^{\prime} 00^{\prime \prime}$ & $29^{\circ} 03^{\prime} 16^{\prime \prime}$ & $82^{\circ} 33^{\prime} 00^{\prime \prime}$ & $82^{\circ} 36^{\prime} 00^{\prime \prime}$ & Dolpa, Jajarkot \\
\hline 23 & Api Naugad HEP & 4.84 & Naugad & $29^{\circ} 48^{\prime} 40^{\prime \prime}$ & $29^{\circ} 49^{\prime} 35^{\prime \prime}$ & $80^{\circ} 40^{\prime} 00^{\prime \prime}$ & $80^{\circ} 41^{\prime} 03^{\prime \prime}$ & Darchula \\
\hline 26 & Aayu Chhatigad HEP & 13.942 & Chatti Gad & $29^{\circ} 48^{\prime} 50^{\prime \prime}$ & $29^{\circ} 49^{\prime} 35^{\prime \prime}$ & $80^{\circ} 52^{\prime} 37^{\prime \prime}$ & $80 \circ 53^{\prime} 10^{\prime \prime}$ & Darchula \\
\hline 27 & Middle Chameliya HEP & 35 & Chameliya Khola & $29^{\circ} 42^{\prime} 55^{\prime \prime}$ & $29^{\circ} 45^{\prime} 00^{\prime \prime}$ & $80^{\circ} 42^{\prime} 38^{\prime \prime}$ & $80^{\circ} 46^{\prime} 45^{\prime \prime}$ & Darchula, Baitadi \\
\hline 28 & Lower Chameliya HEP & 20 & Chameliya & $29^{\circ} 40^{\prime} 00^{\prime \prime}$ & $29^{\circ} 41^{\prime} 40^{\prime \prime}$ & $80^{\circ} 34^{\prime} 41^{\prime \prime}$ & $80^{\circ} 37^{\prime} 59^{\prime \prime}$ & Darchula, Baitadi \\
\hline 29 & Super Lohare Khola -A HEP & 1.3 & Lohare Khola & $28^{\circ} 57^{\prime} 11^{\prime \prime}$ & $28^{\circ} 57^{\prime} 49^{\prime \prime}$ & $81^{\circ} 47^{\prime} 09^{\prime \prime}$ & $81^{\circ} 48^{\prime} 09^{\prime \prime}$ & Dailekh \\
\hline 30 & Super Lohare Khola HEP & 1.53 & Lohare & $28^{\circ} 55^{\prime} 01^{\prime \prime}$ & $28^{\circ} 55^{\prime} 45^{\prime \prime}$ & $81^{\circ} 48^{\prime} 32^{\prime \prime}$ & $81^{\circ} 49^{\prime} 06^{\prime \prime}$ & Dailekh \\
\hline 31 & Upper Rukum Gad HEP & 4.7 & $\begin{array}{l}\text { Rukum Gad (Lukum, } \\
\text { Thaban, Kuchiba }\end{array}$ & $28^{\circ} 34^{\prime} 19^{\prime \prime}$ & $28^{\circ} 35^{\prime} 24^{\prime \prime}$ & $82^{\circ} 37^{\prime} 50^{\prime \prime}$ & $82^{\circ} 38^{\prime} 49^{\prime \prime}$ & Rukum \\
\hline 32 & Sani Bheri HEP & 44.7 & Sani Bheri & $28^{\circ} 38^{\prime} 50^{\prime \prime}$ & $28^{\circ} 40^{\prime} 43^{\prime \prime}$ & $82^{\circ} 39^{\prime} 23^{\prime \prime}$ & $82^{\circ} 42^{\prime} 30^{\prime \prime}$ & Rukum \\
\hline 33 & Sisne Khola HEP & 4.78 & Sisne Khola & $28^{\circ} 41^{\prime} 02^{\prime \prime}$ & $28^{\circ} 42^{\prime} 08^{\prime \prime}$ & $82^{\circ} 41^{\prime} 23^{\prime \prime}$ & $82^{\circ} 42^{\prime} 30^{\prime \prime}$ & Rukum \\
\hline 34 & Syarpu HEP & 3.3 & Dharne & $28^{\circ} 40^{\prime} 10^{\prime \prime}$ & $28^{\circ} 41^{\prime} 25^{\prime \prime}$ & $82^{\circ} 28^{\prime} 24^{\prime \prime}$ & $82^{\circ} 29^{\prime} 25^{\prime \prime}$ & Rukum \\
\hline 35 & Sani Bheri-2 HEP & 23.31 & Sani Bheri & $28^{\circ} 37^{\prime} 30^{\prime \prime}$ & $28^{\circ} 38^{\prime} 52^{\prime \prime}$ & $82^{\circ} 37^{\prime} 02^{\prime \prime}$ & $82^{\circ} 38^{\prime} 50^{\prime \prime}$ & Rukum \\
\hline 36 & Sani Bheri 3 HEP & 49.59 & Sani Bheri & $28^{\circ} 37^{\prime} 15^{\prime \prime}$ & $28^{\circ} 40^{\prime} 15^{\prime \prime}$ & $82^{\circ} 29^{\prime} 48^{\prime \prime}$ & $82^{\circ} 35^{\prime} 15^{\prime \prime}$ & Rukum \\
\hline 37 & Lower Rukumgad HEP & 5.25 & Rukum gad & $28_{\circ} 36^{\prime} 58^{\prime \prime}$ & $28^{\circ} 37^{\prime} 52^{\prime \prime}$ & $82^{\circ} 37^{\prime} 30^{\prime \prime}$ & $82^{\circ} 38^{\prime} 25^{\prime \prime}$ & Rukum \\
\hline 38 & Nalsyau Gad Storage HEP & 417 & Nalsyau & $28^{\circ} 47^{\prime} 21^{\prime \prime}$ & $28^{\circ} 58^{\prime} 00^{\prime \prime}$ & $82^{\circ} 14^{\prime} 00^{\prime \prime}$ & $82^{\circ} 20^{\prime} 00^{\prime \prime}$ & Jajarkot \\
\hline 39 & Bheri-2 HEP & 256 & Bheri & $28^{\circ} 46^{\prime} 45^{\prime \prime}$ & $28^{\circ} 52^{\prime} 00^{\prime \prime}$ & $82^{\circ} 18^{\prime} 41^{\prime \prime}$ & $82^{\circ} 26^{\prime} 40^{\prime \prime}$ & Jajarkot \\
\hline 40 & Taksu Khola HEP & 7.1 & Taksu & $28^{\circ} 52^{\prime} 23^{\prime \prime}$ & $28^{\circ} 53^{\prime} 50^{\prime \prime}$ & $82^{\circ} 26^{\prime} 15^{\prime \prime}$ & $82^{\circ} 27^{\prime} 20^{\prime \prime}$ & Jajarkot \\
\hline 41 & Bheri-1 HEP & 270 & Bheri & $28^{\circ} 52^{\prime} 00^{\prime \prime}$ & $28^{\circ} 57^{\prime} 32^{\prime \prime}$ & $82^{\circ} 26^{\prime} 40^{\prime \prime}$ & $82^{\circ} 31^{\prime} 28^{\prime \prime}$ & Jajarkot, Rukum \\
\hline 42 & Bheri 4 HEP & 300 & Bheri & $28^{\circ} 33^{\prime} 30^{\prime \prime}$ & $28^{\circ} 43^{\prime} 15^{\prime \prime}$ & $81^{\circ} 59^{\prime} 30^{\prime \prime}$ & $82^{\circ} 15^{\prime} 00^{\prime \prime}$ & $\begin{array}{l}\text { Jajarkot, Rukum, } \\
\text { Salyan, Surkhet }\end{array}$ \\
\hline 43 & Madi Storage HEP & 156 & Madi & $28^{\circ} 13^{\prime} 30^{\prime \prime}$ & $28^{\circ} 20^{\prime} 55^{\prime \prime}$ & $82^{\circ} 33^{\prime} 40^{\prime \prime}$ & $82^{\circ} 39^{\prime} 15^{\prime \prime}$ & Rolpa \\
\hline 44 & Phukot Karnali HEP & 426 & Karnali & $29^{\circ} 09^{\prime} 32^{\prime \prime}$ & $29^{\circ} 19^{\prime} 03^{\prime \prime}$ & $81^{\circ} 35^{\prime} 28^{\prime \prime}$ & $81^{\circ} 45^{\prime} 00^{\prime \prime}$ & Kalikot \\
\hline 45 & $\begin{array}{l}\text { Upper Ruru Banchu Khola } \\
\text { HEP }\end{array}$ & 24.5 & Rurubanchu Khola & $29^{\circ} 05^{\prime} 00^{\prime \prime}$ & $29^{\circ} 06^{\prime} 30^{\prime \prime}$ & $81^{\circ} 48^{\prime} 13^{\prime \prime}$ & $81^{\circ} 51^{\prime} 35^{\prime \prime}$ & Kalikot \\
\hline \multirow[t]{2}{*}{46} & Thuligad Khola Small HEP & 9 & Thuli Gad & $28^{\circ} 55^{\prime} 50^{\prime \prime}$ & $28^{\circ} 58^{\prime} 47^{\prime \prime}$ & $80^{\circ} 47^{\prime} 41^{\prime \prime}$ & $80^{\circ} 51^{\prime} 22 "$ & Doti, Kailali \\
\hline & Total & 6923.982 & & & & & & \\
\hline
\end{tabular}


completed, while $53 \mathrm{~km}$ eastern canal construction is underway (Investopaper 2020). Besides these, several farmers widely used small irrigation canals (called Kulo) from nearby small rivers and streams and managed themselves (Poudel \& Sharma 2012).

\section{2 | Biodiversity}

The freshwater biodiversity of Nepal is quite diverse and supports a high diversity of wild flora and fauna and other hydrofauna (Lamsal et al. 2014). It is also a habitat of several globally threatened species of mammals, birds, reptiles, amphibians, fish, and other macro-invertebrates. This rich diversity is possibly explained by the wide elevation distribution of various aquatic ecosystems such as rivers, streams, high altitude lakes, and ponds from hot and humid to cold alpine climates.

\section{Birds}

Among 886 bird species in Nepal (DNPWC \& BCN 2018), 200 species (22 percent) are known to be dependent on wetlands (Inskipp et al. 2017). The BirdLife International (2019) listed 38 species which are globally threatened species that are wetlanddependent, including pink-headed duck (Rhodonessa caryophyllacea), greater adjutant (Leptoptilos dubius), lesser adjutant (Leptoptilos javanicus), sarus crane (Grus antigone), black-necked crane (Grus nigricollis), lesser florican (Sypheotides indicus), Baikal teal (Anos formosa), swamp francolin (Francolinus gularis), Baer's pochard (Aythya baeri), spot-billed pelican (Pelecanus philippensis), Indian skimmer (Rynchops albicollis), and Pallas's fish-eagle (Haliaeetus leucoryphus).

Western Nepal also supports the high richness of avifauna (Baral \& Inskipp 2009). Shukla Phanta National Park provides habitat for more than 450 bird species; among them, 23 species are globally threatened, and 74 species are nationally threatened (Poudyal \& Chaudhary 2019). The park also has several streams and lakes. Rani Lake is the largest lake in the Park and supports many globally threatened bird species such as black stork (Ciconia nigra), black bittern (Dupetor flavicollis), yellow bittern (Ixobrychus sinensis), and striated grassbird (Megalurus palustris). Eight species of wetland birds were recorded from Ramaroshan Lake, including globally vulnerable Asian Woollyneck and five migratory birds (Poudel \& Adhikari 2020). Similarly, 241 species of birds, including 49 wetland bird species, were recorded from Rara National Park. Rara Lake supports the feeding and breeding grounds for migratory and residential wetland-dependent birds (DNPWC 2019). The Pokshundo Lake supports more than 46 species of wetland birds (DNPWC 2020c). Acharya and Ghimirey (2015) recorded 114 species of birds in the area between Limi Valley, including notable wetland birdscommon tern (Sterna hirundo) and ibisbill (Ibidorhyncha struthersii). The biodiversity study of four lakes (Sati Karnali, Jhilmila, Rani, and Ramaroshan) reported 41 wetland birds from Sati Karnali, 15 species from Jhilmila, 48 species from Rani Lake, and 15 species from Ramaroshan Lake (Khatiwada et al. 2019a)
(Table 5). Similarly, Karnali and Mahakali rivers also provide a suitable habitat for various water birds (Manel et al. 2000).

Table 5. Checklist of globally threatened wetland birds recorded by various authors from Western Nepal (Here, EN-Endangered, VU-Vulnerable, NT-Near Threatened)

\begin{tabular}{|c|c|c|c|}
\hline SN & Common Name & Scientific Name & $\begin{array}{l}\text { IUCN } \\
\text { Status }\end{array}$ \\
\hline 1 & Pink-headed duck & Rhodonessa caryophyllacea (Latham, 1790) & CR \\
\hline 2 & Baer's pochard & Aythya baeri (Radde, 1863) & CR \\
\hline 3 & Pallas's fish-eagle & Haliaeetus leucoryphus (Pallas, 1771) & EN \\
\hline 4 & Lesser florican & Sypheotides indicus (Miller, 1782) & EN \\
\hline 5 & Indian skimmer & Rynchops albicollis (Swainson, 1838) & EN \\
\hline 6 & Lesser adjutant & Leptoptilos javanicus (Horsfield, 1821) & VU \\
\hline 7 & Common pochard & Aythya ferina (Linnaeus, 1758) & VU \\
\hline 8 & Asian woollyneck & Ciconia episcopus (Boddaert, 1783) & NT \\
\hline 9 & Sarus crane & Antigone antigone (Linnaeus, 1758) & VU \\
\hline 10 & Lesser fish-eagle & Icthyophaga humilis (Müller \& Schlegel, 1841) & NT \\
\hline 11 & River lapwing & Vanellus duvaucelii (Lesson, 1826) & NT \\
\hline 12 & Painted stork & Mycteria leucocephala (Pennant, 1769) & NT \\
\hline 13 & Oriental darter & Anhinga melanogaster Pennant, 1769 & NT \\
\hline 14 & Spot-billed pelican & Pelecanus philippensis Gmelin, 1789 & NT \\
\hline 15 & Northern lapwing & Vanellus vanellus (Linnaeus, 1758) & NT \\
\hline 16 & Black-necked crane & Grus nigricollis Przevalski, 1876 & NT \\
\hline
\end{tabular}

\section{Mammals}

A total of 212 species of mammals have been recorded from Nepal (Amin et al. 2018). Among them, only 27 mammalian faunae are under the protection of the NPWC Act 1973 (DNPWC 2020b). The western low-land of Nepal supports the key globally threatened mammals, and most of them are wetland-dependent. These include tiger (Panthera tigris), pygmy hog (Sus salvanius); hispid hare (Caprolagus hispidus), South Asian river dolphin (Platanista gangetica), greater one-horned rhinoceros (Rhinoceros unicornis), elephant (Elephas maximus) and barasingha (Cervus duvaucelii) (Henshaw 1994; Pradhan et al. 2008; Jnawali et al. 2011; Aryal et al. 2012; Flagstad et al. 2012).

Karnali River is a major habitat for globally endangered South Asian river dolphin in Nepal. Shrestha (1989) reported 12 individuals of dolphin from Patharboji, Lalmati, and the area upstream of Golaghat up to Kachali of Karnali River. Later, Smith (1994) recorded only seven individuals of dolphin, whereas Timilsina et al. (2003) reported only four individuals from Karnali River. Based on the data, there is decreasing trend of population of the Dolphin in the Karnali River $\left(y=-1.2238 x+16.288, R^{2}=\right.$ 0.4383 ) (Fig. 5). Dolphins prefer slow water current with adequate amounts of deep pool habitats in Karnali River (Paudel et al. 2015a). Paudel et al. (2015a) estimated 12 dolphins in the two southern sections of the Karnali River. Shah et al. (2020a) reported 43 dolphins from Mohana River and its tributaries but not recorded from Karnali River.

River geometry and infrastructure development (constructed dam at the India/Nepal border of Karnali River) caused the greatest threats to dolphins due to changes in river flow characteristics (Paudel et al. 2015b). Anthropogenic activities in the river such as the presence of fishing boats, stone quarries, and motorboats 
used for local transportation, construction of irrigation canals, extraction of construction materials such as sands, gravels, stones from the river are the other major threats to Dolphins (Malla 2009; Khanal et al. 2016). Furthermore, changing the natural course of river systems (e.g., Geruwa to western flow in Karnali) fluctuate the water depth of the river. The deep pools are critical habitats that greatly affect the occurrence and distribution of dolphins (WWF 2006; Khanal et al. 2016).

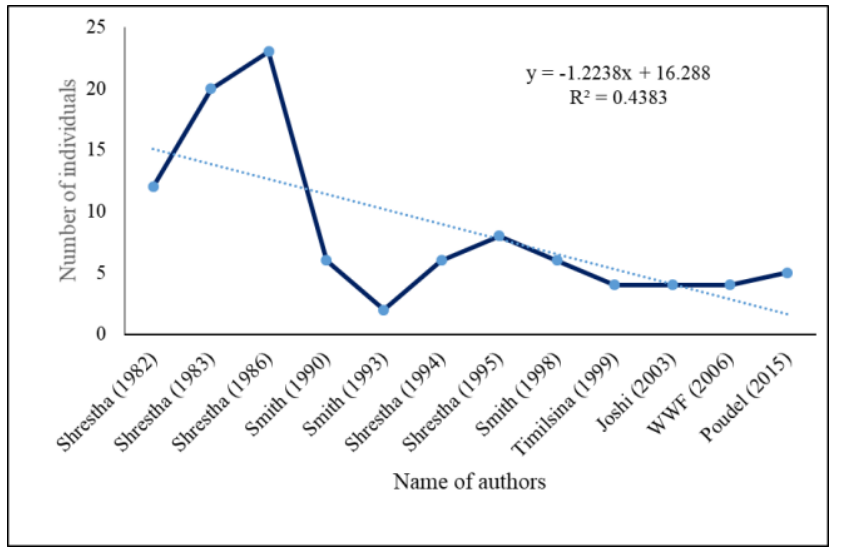

Figure 5. Population trend of the dolphin. (Source: WWF 2006 and Paudel et al. 2015b).

\section{Fish}

Nepal is very rich in native fish diversity. There are more than 186 indigenous and 11 introduced fish taxa belonging to 93 Genera from 31 Families and 11 Orders (Shrestha 1990, Shrestha 1999; Ng \& Edds 2005; Edds 2007; Edds \& Ng 2007; Shrestha \& Edds 2012). Literature show that about 121 species of fishes are found in the Karnali River and its tributaries. Similarly, Rara Lake provides a habitat for three endemic fishes named Schizothorax macrophthalmus, $S$. nepalensis, and $S$. raraensis (Terashima 1984; Acharya \& Paudel 2020). Lamsal et al. (2014) reported 19 fish species from Ghodaghodi Lake, Kailali district, whereas DoF (2017) reported more than 29 species from the same area. These different statistics of fish diversity could be due to the difference in data collection techniques/sampling time used in these studies. Most of these studies were carried out through the questionnaire survey and anecdotal information; therefore, a rigorous field-based study is needed to explore the current change in diversity pattern. Khatri et al. (2019) recorded 16 species of fishes from the Bheri and its tributaries followed by 22 species from Babai and its tributaries, including three species, viz., Neolissochilus hexagonolepis, Schizothorax richardsonii and Tor putitora as globally threatened fish. Khatiwada et al. (2019a) reported 29 species of fishes from Sati Karnali Lake, three species from Jhilmila Lake, six species from Rani Lake, and three species, namely 'Bucche Asala' (Schizothorax nepalensis), 'Tikhe Asala' (Schizothorax richardsonii), and garra (Garra gotyla) from Ramaroshan Lake. Still, information regarding the current conservation status of wetlands fishes of low-land, western Nepal is greatly lacking.

\section{Herpetofauna}

Nepal supports a rich unrecognized amphibian and reptilian diversity (Schleich \& Kästle 2002). It harbors more than 54 species (Khatiwada et al. 2020, Khatiwada et al. 2021) of amphibians and 134 taxa/species of reptiles (Shah \& Tiwari 2004), but information about them is generally derived from relatively old studies (Smith \& Battersby 1953; Nanhoe \& Ouboter 1987; Mitchell \& Zug 1995; Zug \& Mitchell 1995). These records are primarily based on the species already discovered from neighboring countries like India and Tibet in China. However, these numbers are not based on detailed field studies of population densities within Nepal but from presence-only surveys or anecdotal information. This confirmed that Nepalese herpetofauna are poorly understood and many of them are incorrectly classified (Khatiwada 2017; Khatiwada et al. 2017). In western Nepal, in the late 1970s, French herpetologist A. Dubois made a significant contribution to the biology of Nepalese amphibians. He and other collaborators collected more than 15,000 amphibian specimens from Nepal (Dubois 1999) and described more than 10 new species (Dubois 1973, 1974, 1974 1975a, 1975b, 1977; Dubois \& Matsui 1983; Dubois 1984, 1987). In the meantime, Nanhoe and Ouboter (1987) surveyed the amphibians and reptiles of the Annapurna-Dhaulagiri region of western Nepal and compiled an updated checklist based on both field observation and museum specimens and they reported 42 species of herpetofauna. Similarly, Khatiwada et al. (2019a) reported seven species of amphibian and eight species of reptiles from Sati Karnali Lake area; likewise, 10 species of amphibian and 16 species of reptiles from the Rani Lake area, five species of reptiles and seven species of amphibian from Ramaroshan area, six species of amphibian and seven species of reptiles from Jhilmila Lake area. Recently, Khatiwada et al. (2020) described a new species of Amolops frogs, which has wide distributional ranges in the mid-hill of Darchula, the Mahakali River basin of western Nepal. Skittering frog (Euphlyctis cyanophlyctis), Indian bull frog (Hoplobatrachus tigerinus), marbled toad (Duttaphrynus stomaticus), Syhadra frog (Minervarya syhadrensis) from lowland, black-spined toad (Duttaphrynus melanosticus), common Indian tree frog (Polypedates maculatus) from Midhill and Himalayan toad (Duttaphrynus himalayanus), Liebig's paa frog (Nanorana legibii), small paa frog (Nanorana minica) from highland were reported from of Karnali and Mahakali river basins (Khatiwada et al. 2019).

\section{Aquatic invertebrates}

Macroinvertebrates are major fauna in the freshwater ecosystem and have been widely used as a proxy/indicator of wetland conditions based on species richness, composition, or functional diversity (Ormerod et al. 1994; Marzin et al. 2012). Unfortunately, current knowledge of benthic macroinvertebrates in the freshwater ecosystem in Nepal is still very fragmentary (Brewin \& Ormrod 1994; Suren 1994; Manel et al. 2000). In western Nepal Himalaya, only a few studies have assessed richness, diversity, and relationship with environmental variables using 
benthic macroinvertebrate communities as the model organisms (Shah 2020; Matangulu et al. 2017; Suren 1994; Manel et al. 2000). Suren (1994) examined the relationship of elevation and the land-use changes on the richness and composition of macroinvertebrates in the Karnali River. Manel et al. (2000) reported the curvilinear relationship of macroinvertebrates with elevation. Major invertebrate fauna in the Karnali River was Orders Ephemeroptera, Plecoptera, Trichoptera, and Diptera. Budha (2010) made an inventory of Barhaiya Lake, Bardiya and reported eight species of freshwater snails and mussels- Pila globosa, Bellamya bengalensis, Indoplanorbis exustus, Melanoides tuberculatus, Lymnaea acuminata, Gyraulus sp., Segmentina sp. and Lamellidens marginalis. A study of the macroinvertebrates in West Seti river basin recorded 34 Families of the macroinvertebrates belonging to 7 Orders (Matangulu et al. 2017). Similarly, Shah et al. (2020b) reported 128 taxa of macroinvertebrates belonging to 84 families and 22 orders from the Karnali River Basin.

\section{Wetland associated vegetation}

Nepal's wetland harbors a wide range of vegetation. A recent publication listed over 711 species of plants including ferns as wetland plants (Ghimire et al. 2020); however, their list may contain some of the forest flora. Terai wetlands are rich in plant diversity. Sah et al. (2002) carried out a floristic inventory of Ghodaghodi Lake and reported a total of 401 plant species belonging to 264 Genera and 84 Families, among them 269 were dicots, and 132 were monocots. Lamsal et al. (2014) found 45 species of aquatic plants; among them, nine were submerged, six free-floating, 21 floating leaf, and nine emergent species from the same area. Mahakali River and associated wetlands also support high plant diversity. The study by Kunwar et al. (2015) from the far western region found 140 plant species, with 78 species having ethnomedicinal importance. Similarly, the study of Khatiwada et al. (2019a) reported 37 species of aquatic macrophytes from Sati Karnali Lake followed by 15 species from Jhilmila Lake, 28 species from Rani Lake, and 30 species from Ramaroshan Lake complex.

\section{4 | Threats to freshwater ecosystems}

About $5.41 \%$ of the total coverage of wetlands of Nepal was decreased due to the expansion of croplands in Nepal ( $\mathrm{Li}$ et al. 2017; MoFE 2018). The major drivers of degradation of wetlands are population growth, more demand for wetland resources, removal of construction materials from the wetlands, invasions of alien species, siltation, and unclear national policies and management issues (MoFE 2018; NLCDC 2020). Besides these, fishing, grazing, siltation, pollution, extraction of construction materials nearby lakes, and rivers and conversion of wetlands for farming practices are the major threats to the aquatic ecosystems of Nepal.

The introduction of invasive species (commercial fishes), the use of fertilizers and pesticides further deteriorate the water health of wetlands (Budha 2010). Wetlands are shrinking, particularly in the lowland areas, because of siltation and eutrophication. In Terai, the majority of ox-bow lakes have been facing the problems of siltation and plant succession; for example, Ghodaghodi Lake Complex, Rani Lake and Jhilmila Lake are dominated by emergent and amphibious vegetation (Baral \& Inskipp 2009; Budha 2010; Lamsal et al. 2014). Over-exploitation of aquatic biodiversity also imposes a serious threat to aquatic biodiversity. Amphibians and reptiles have long been used by humans as food and medicine (Gonwouo \& Rödel 2008; Khatiwada \& Haugaasen 2015). Studies have shown that frogs are an important source of livelihood for many people (Khatiwada \& Haugaasen 2015) and remain an integral part of local medicinal heritage (Mohneke et al. 2011). Particular species are collected on a large scale, and over-collecting may lead to local extinctions or severe population declines. Quasipaa frogs in China have become rare, some being even on the verge of extinction, due to over-exploitation (Chan et al. 2014). As many amphibian species predictably aggregate for reproduction or hibernation, this makes them particularly vulnerable to intensive collecting efforts. Recent studies have indicated that commercial or subsistence harvesting has contributed to a decline in many reptile species (Webb et al. 2002). It has been revealed that Paa and Amolops are the most exploited frog species by the local people for food and medicinal purposes in mountainous parts of Nepal (Khatiwada \& Haugaasen 2015; Khatiwada et al. 2019b; Khatiwada et al. 2020). This heavy exploitation may also lead to local or global declines and even extinctions through the unsustainable collection (Warkentin et al. 2009). Monitoring of these species and collection activities is, therefore, a conservation priority in the study area and elsewhere in Nepal.

\section{5 | Conservation history of wetlands' exploration in}

\section{Nepal}

Nepal has a long conservation history of wetland, particularly related to the protection of nature and its natural resources. The first Wildlife Act was introduced in 1957 for the conservation of the Rhinoceros population in Chitwan. Later, in 1964, a rhino sanctuary was declared in Chitwan district (now Chitwan National Park), and a special guard force called the Gaida Gasti (Rhino Patrol) was created in the same year (Heinen \& Kattel 1992). Since then, several conservation efforts have been undertaken for the future conservation of forests and overall biodiversity. The Government of Nepal launched the National Parks and Wildlife Conservation Act in 1973 (in short, it is called NPWC Act 1973), with national commitments for the conservation of protected areas and important wildlife species (HMG 1973). Nepal showed its conservation commitment with the Ramsar convention by signing on April 17, 1988. As a response to Ramsar signatory members, Nepal declared the Koshi Tappu Wildlife Reserve (KTWR) as the first Ramsar site of Nepal in 1988 (IUCN Nepal 2004; NLCDC 2019). To date, ten wetland sites of Nepal have been listed in the Ramsar sites (NLCDC 2019). 
IUCN (1998) made the first wetland inventory and provided the conservation status of wetlands of low land Terai, Nepal. Then several surveys and inventories of the wetlands of Nepal have been undertaken (IUCN 1998; WECS 2003; IUCN Nepal 2004; NLCDC 2019), and these studies highlighted the major problems facing the wetlands and status of wetlands of Nepal. National Wetland Policy 2003 highlighted the need for an inventory of wetland resources. This policy aimed to conserve and manage wetlands resources wisely and in a sustainable way with local people's participation (GoVN 2003; NLCDC 2019). Hence, National Lake Conservation Development Committee (NLCDC) focuses on the conservation of lakes and preparing the lake database of Nepal. Recently, the Department of Forests and Soil Conservation, Government of Nepal has published a report on the Wetland of Western Nepal and has made an inventory of lakes of western and far-western Nepal (DoF 2017). They recognized a total of 92 lakes/wetlands from 13 districts. Among them, the Kailali district occupied the largest number of lakes ( $\mathrm{n}$ $=48$ ), followed by Kanchanpur $(n=28)$ and Achham $(n=7)$ (DoF 2017). Many rules and regulations were made to manage wetland resources, but its implementation is very weak. Many Acts and Rules are contradictory to each other. The policy of the DNPWC act, the Forest Act, is in contradiction with National Wetland Policy. Hence, these gaps should be minimized.

\section{6 | Conclusion}

Freshwater ecosystems of western Nepal (Karnali and Mahakali river basins) support habitats for several threatened and endangered flora and fauna and are distributed from low to higher elevation in various climatic zones. Wetlands of western Nepal are rich in biological diversity and provide a wide range of goods and ecosystem services, including irrigation, domestic supply, fisheries, and recreation to humankind. Fishing, grazing, and conversion of wetlands for farming practices are the major threats to the aquatic ecosystem of western Nepal. There are several acts and regulations related to wetland conservation and management; this sector has received inadequate attention in the national policy. Available studies on freshwater/wetland research and conservation initiatives in western Nepal assessed richness, diversity of wetland fauna and relationship with limnological factors. However, the effect of current land-use, climate change and socio-economic factors leading to limnological changes have not been explored adequately. Further, policy gaps in freshwater management have received limited attention in conservation. Therefore, further research on the effect of current land use, socioeconomic, and policy factors influencing wetland biodiversity and sustainability is suggested.

\section{Acknowledgements}

We would like to thank the USAID Pani Program for the financial support. We are obliged for the contributors, authors and publishers who provided us valuable information.

\section{Authors' contributions}

Khatiwada, J. R. and Adhikari, J. N. conceptualized the study, collected the literatures and prepared the manuscript. All authors finalized the manuscript with their inputs.

\section{Conflicts of interest}

Authors declare no conflict of interest.

\section{ORCID}

Janak Raj Khatiwada (D) https://orcid.org/0000-0003-1507-2288 Jagan Nath Adhikari (iD https://orcid.org/0000-0002-6881-6618 Lila Nath Sharma (iD https://orcid.org/0000-0001-5939-3118

\section{References}

Abell, R., Thieme, M. and Lehner, B. 2011. Indicators for assessing threats to freshwater biodiversity from humans and humanshaped landscapes. In: Cincotta R., Gorenflo L. (Eds.) Human population: its influences in biological divrsity. Springer, Berlin, Heidelberg, pp 103-124. https://doi.org/10.1007/978-3-64216707-2_7

Acharya, K. P. and Paudel, P. K. 2020. Biodiversity in Karnali Province: current status and conservation. Ministry of Industry, Tourism, Forest and Environment, Karnali Province Government, Surkhet, Nepal.

Allen, D. J., Molur, S. and Danie, B. A. 2010. The status and distribution of freshwater biodiversity in the Eastern Himalaya. IUCN (International Union for Conservation of Nature) and Zoo Outreach Organisation, Cambridge, UK, Gland, Switzerland and Coimbatore, India.

Amin, R., Baral, H. S., Lamichhane, B. R., Poudyal, L. P., Lee, S., Jnawali, S. R. et al. 2018. The status of Nepal's mammals. Journal of Threatened Taxa 10:11361-11378.

https://doi.org/10.11609/jott.3712.10.3.11361-11378

Aryal, A., Brunton, D., Ji, W., Yadav, H. K., Adhikari, B. and Raubenheimer, D. 2012. Diet and Habitat use of Hispid Hare Caprolagus hispidus in Shuklaphanta Wildlife Reserve, Nepal. Mammal Study 37:147-154.

https://doi.org/10.3106/041.037.0205

Atkinson, C. L., Capps, K. A., Rugenski, A. T. and Vanni, M. J. 2017. Consumer-driven nutrient dynamics in freshwater ecosystems: from individuals to ecosystems. Biological Reviews 92:20032023. https://doi.org/10.1111/brv.12318

Baillie, J. E., Collen, B., Amin, R., Akcakaya, H. R., Butchart, S. H., Brummitt, N. et al. 2008. Toward monitoring global biodiversity. Conservation Letters 1:18-26. https://doi.org/10.1111/j.1755263X.2008.00009.x

Baral, H. and Inskipp, C. 2009. The birds of Sukla Phanta Wildlife Reserve, Nepal. Our Nature 7:56-81.

Bell, D. E. 1992. The 1992 convention on biological diversity: the continuing significance of US objections at the Earth Summit. George Washington Journal of International Law and Economics 26:479-537. 
Bhatt, R. P. 2017. Hydropower development in Nepal- climate change, impacts and implications. Renewable Hydropower Technologies. 28:75-98. https://doi.org/10.5772/66253

Bhuju, U. R., Khadka, M., Neupane, P. K. and Adhikari, R. 2010. A map based inventory of lakes in Nepal. Nepal Journal of Science and Technology 11:173-180. https://doi.org/10.3126/njst.v11i0.4141

Bhuju, U. R., Shakya, P. R., Basnet, T. B. and Shrestha, S. 2007. Nepal biodiversity resource book: protected areas, Ramsar sites, and World Heritage sites. ICIMOD / MOEST-GoN / UNEP, Kathmandu, Nepal.

BirdLife International. 2019. Country profile: Nepal. Cambridge, UK, BirdLife International.

Bogardi, J. J., Leentvaar, J. and Sebesvári, Z. 2020. Biologia Futura: integrating freshwater ecosystem health in water resources management. Biologia Futura 71:337-358. https://doi.org/10.1007/s42977-020-00031-7

Brewin, P. A. and Ormrod, S. J. 1994. Macroinvertebrate drift in streams of the Nepalese Himalaya. Freshwater Biology 32:573583. https://doi.org/10.1111/j.1365-2427.1994.tb01148.x

Budha, P. B. 2010. Inventory of wetland fauna and sustainable resource utilization of Bahraiya Lake, Bardia, Mid-western Nepal: Issues and future prospects. TIGERPAPER, Regional Quarterly Bulletin on Wildlife and National Park Management 37:10-20.

Byers, A. 2005. Contemporary human impacts on Alpine ecosystems in the Sagarmatha (Mt. Everest) national park, Khumbu, Nepal. Annals of the Association of American Geographers 95:112140. https://doi.org/10.1111/j.1467-8306.2005.00452.x

Chalaune, T. B., Dangol, A., Sharma, J. and Sharma, C. M. 2020. First results on physico-chemical status and bathymetry of lakes in Ramaroshan Wetland, Far-West Nepal. Nepal Journal of Environmental Science 8:17-27. https://doi.org/10.3126.njes.v8i1.34464

Chan, H. K., Shoemaker, K. T. and Karraker, N. E. 2014. Demography of Quasipaa frogs in China reveals high vulnerability to widespread harvest pressure. Biological Conservation 170:3-9. https://doi.org/10.1016/j.biocon.2013.12.014

Collen, B., Whitton, F., Dyer, E. E., Baillie, J. E., Cumberlidge, N., Darwall, W. R., et al. 2014. Global patterns of freshwater species diversity, threat and endemism. Global Ecology and Biogeography 23:40-51. https://doi.org/10.1111/geb.12096

DNPWC. 2019. Rara national park and its buffer zone management plan 2076/77-2080/81. Department of National Parks and Wildlife Conservation Nepal.

DNPWC. 2020a. National parks, wildlife reserves and conservation areas of Nepal. Department of National Parks and Wildlife Conservation (DNPWC), Kathmandu, Nepal.

DNPWC. 2020b. Protected mammals of Nepal. Department of National Parks and Wildlife Conservation (DNPWC), Kathmandu.

DNPWC. 2020c. Shey-Phoksundo National Park. Department of National Parks and Wildlife Conservation.

DNPWC and BCN. 2018. Birds of Nepal: An official checklist. Department of National Parks and Wildlife Conservation, Bird Conservation Nepal.

Dobremez, J. F. 1976. Le Népal: Écologie et Biogéographie. Paris : Centre National de la Recherche Scientifique.
DoED. 2020. Hydro-power in Nepal. Ministry of Energy, Water Resources and Irrigation, Government of Nepal, Department of Electricity Development.

DoF. 2017. Wetlands of western Nepal: A brief profile of selected lakes. Department of Forests, Government of Nepal.

DoFD. 2011. Annual Progress Report 2011. Directorate of Fisheries Development (DoFD), Kathmandu.

Dubois, A. 1973. Diagnoses of 3 new species of amphibia from Nepal. Bulletin de la societe zoologique de france-evolution et zoologie 98:495-497.

Dubois, A. 1974a. Research on zoogeography of Nepalese amphibians. Pages 788-791 in Bulletin De La Societe Zoologique De France-Evolution Et Zoologie. Soc Zoologique France Inst Oceanographique 195 Rue Saint-Jacques, 75005 Paris, France, Paris, France.

Dubois, A. 1975a. Un nouveau sous-genre (Paa) et trois nouvelles espèces du genre Rana. Remarques sur la phylogénies des Ranidés (Amphibiens, Anoures). Comptes Rendus Hebdomadaires des Séances de l'Académie des Sciences. Paris Serie 3, Zoologie 324:1093-1115.

Dubois, A. 1975b. Une nouveau complexe d'espèces jumelles distinguées par le chant: les grenouilles de Népal voisines de Rana limnocharis Boie (Amphibienes, Anoures). Comptes Rendus Hebdomadaires des Séances de l'Académie des Sciences Paris 281:1717-1720.

Dubois, A. 1977. Chants et écologie chez les Amphibiens du Népal. Pages 109-118 in Himalaya Ecolethnologie Colloq Int CNRS.

Dubois, A. 1984. Note preliminaire sur le groupe de Rana limnocharis Gravenhorst, 1829 (Amphibiens, Anoures). Alytes Paris 143159.

Dubois, A. 1987. Miscellanea taxinomica batrachologica. Alytes (Paris) 5:7-95.

Dubois, A. 1999. South Asian Amphibia: a new frontier for taxonomists. Journal of South Asian Natural History 4:1-11.

Dubois, A. and Matsui, M. 1983. A new species of frog (genus Rana, subgenus $\mathrm{Paa}$ ) from western Nepal (Amphibia: Anura). Copeia 1983:895-901.

Dudgeon, D., Arthington, A. H., Gessner, M. O., Kawabata, Z.-I., Knowler, D. J., Lévêque, C. et al. 2006. Freshwater biodiversity: importance, threats, status and conservation challenges. Biological Reviews 81:163-182. https://doi.org/10.1017/S1464793105006950

Edds, D. R. 2007. Fishes in Nepal: ichthyofaunal surveys in seven nature reserves. Ichthyological Exploration of Freshwaters 18:277-287.

Edds, D. R. and Ng, H. H. 2007. Additions to the ichthyofauna of Nepal, with a redescription of Neoeucirrhichthys maydelli (Teleostei: Cobitidae). Ichthyological Exploration of Freshwaters 18:125-132.

Flagstad, Ø., Pradhan, N. M., Kvernstuen, L. G. and Wegge, P. 2012. Conserving small and fragmented populations of large mammals: non-invasive genetic sampling in an isolated population of Asian elephants in Nepal. Journal for Nature Conservation 20:181-190. https://doi.org/10.1016/j.jnc.2012.01.006

Geist, J. 2011. Integrative freshwater ecology and biodiversity conservation. Ecological Indicators 11:1507-1516. https://doi.org/10.1016/j.ecolind.2011.04.002 
Ghimire, M. D., Dhakal, K. S. and Saud, D. S. 2020. A checklist of wetland flora reported from Nepal. Department of plant resources Kathmandu, Nepal.

Gonwouo, L. and Rödel, M. 2008. The importance of frogs to the livelihood of the Bakossi people around Mount Manengouba, Cameroon, with special consideration of the Hairy Frog, Trichobatrachus robustus. Salamandra 44:23-34.

GoVN. 2003. National Wetlands Policy, 2059 (2003) Government of Nepal, Law commission, Kathmandu.

Grytnes, J. A. 2003. Species - richness patterns of vascular plants along seven altitudinal transects in Norway. Ecography 26:291300. https://doi.org/10.1034/j.1600-0587.2003.03358.x

Heinen, J. T. and Kattel, B. 1992. A review of conservation legislation in Nepal: Past progress and future needs. Environmental Management 16:723. https://doi.org/10.1007/BF02645662

Henshaw, J. 1994. The barasingha, or swamp deer, in Suklaphanta Wildlife Reserve, Nepal. Oryx 28:199-206.

https://doi.org/10.1017/S0030605300028568

HMG. 1973. National Parks and Wildlife Conservation Act 2029. Nepal Gazette 2029-11-28 B.S. Department of Forest, Government of Nepal, Kathmandu, Nepal.

ICIMOD. 2014. Rivers of Nepal: Database. International Centre for Integrated Mountain Development (ICIMOD), Kathmandu, Nepal.

Inskipp, C., Baral, H. S., Inskipp, T., Khatiwada, A. P., Khatiwada, M. P., Poudyal, L. P. et al. 2017. Nepal's National red list of birds. Journal of Threatened Taxa 9:9700-9722.

Investopaper. 2020. Sikta irrigation project starts irrigation, project partially completed. Investopaper. MGI Group Pvt. Ltd.

IUCN. 1998. An inventory Nepal's wetlands. IUCN Nepal.

IUCN Nepal. 2004. A review of the status and threats to wetlands in Nepal.

Ives, J. 2004. Himalayan perceptions: Environmental change and the well-being of mountain peoples. Routledge, London, UK.

Jnawali, S., Baral, H., Lee, S., Acharya, K., Upadhyay, G., Pandey, M. et al. 2011. The status of Nepal Mammals: The national red list series. Department of National Parks and Wildlife Conservation, Kathmandu, Nepal.

Junk, W. J., An, S., Finlayson, C., Gopal, B., Květ, J., Mitchell, S. A. et al. 2013. Current state of knowledge regarding the world's wetlands and their future under global climate change: a synthesis. Aquatic Sciences 75:151-167. https://doi.org/10.1007/s00027-012-0278-z

Khadka, N., Zhang, G. and Thakuri, S. 2018. Glacial lakes in the Nepal Himalaya: Inventory and decadal dynamics (1977-2017). Remote Sensing 10:1913. https://doi.org/10.3390/rs10121913

Khanal, G., Suryawanshi, K. R., Awasthi, K. D., Dhakal, M., Subedi, N., Nath, D. et al. 2016. Irrigation demands aggravate fishing threats to river dolphins in Nepal. Biological Conservation 204:386-393. https://doi.org/10.1016/j.biocon.2016.10.026

Khatiwada, J. R. 2017. Integrative taxonomy, diversity, and conservation of Amphibians in the central and eastern Nepal Himalaya. University of Chinese Academy of Sciences, Beijing, China.

Khatiwada, J. R., Adhikari, J. N., Adhikari, D., Sapkota, S., Ghimire, S. R., Budha, P. B. et al. 2019a. Assessment and conservation status of aquatic biodiversity in lower Karnali and Mahakali River basin. Forest Action Nepal, USAID Pani Program.
Khatiwada, J. R. and Haugaasen, T. 2015. Anuran species richness and abundance along an elevational gradient in Chitwan, Nepal. Zoology and Ecology 25:110-119.

Khatiwada, J. R., Shu, G. C., Bin, W., Zhao, T., Xie, F. and Jiang, J. 2020. Description of a new species Amolops Cope, 1865 (Amphibia: Ranidae) from Nepal and nomenclatural validation of Amolops nepalicus Yang, 1991. Asian Herpetological Research 11 (2):1-40. https://doi.org/10.16373/j.cnki.ahr.190052

Khatiwada, J. R., Shu, G. C., Wang, S. H., Thapa, A., Wang, B. and Jiang, J. P. 2017. A new species of the genus Microhyla (Anura: Microhylidae) from Eastern Nepal. Zootaxa 4254:221-239. https://doi.org/10.11646/zootaxa.4254.2.4

Khatiwada, J. R., Wang, B., Zhao, T., Xie, F. and Jiang, J. 2021. An integrative taxonomy of Amphibians of Nepal: an updated status and distribution. Asian Herpetological Research 13:1-35.

Khatiwada, J. R., Zhao, T., Chen, Y., Wang, B., Xie, F., Cannatella, D. C. et al. 2019b. Amphibian community structure along elevation gradients in eastern Nepal Himalaya. BMC Ecology 19:19. https://doi.org/10.1186/s12898-019-0234-z

Khatri, K., Jha, B. R., Gurung, S., Khadka, U. R., Pokharel, S., Adhikari, A. et al. 2019. Fish diversity and distribution status in Bheri and Babai River, Mid-Western, Nepal. Pages 206-212 in International Conference on Natural Resources, Agriculture and Society in Changing Climate. Kathmandu University, Kathmandu.

Kunwar, R. M., Baral, K., Paudel, P., Acharya, R. P., Thapa-Magar, K. B., Cameron, M. et al. 2016. Land-use and socioeconomic change, medicinal plant selection and biodiversity resilience in Far Western Nepal. PLoS One 11:e0167812.

Kunwar, R. M., Pyakurel, P., Burlakoti, C., Pyakurel, P. and Shah, S. G. 2015. Phyto-ecological assessment of Mahakali River, Farwestern Nepal. Journal of Natural History Museum 29:32-48.

Lacoul, P. and Freedman, B. 2005. Physical and chemical limnology of 34 lentic waterbodies along a tropical-to-alpine altitudinal gradient in Nepal. International Review of Hydrobiology 90:254276. https://doi.org/10.1002/iroh.200410766

Lamsal, P., Pant, K., Kumar, L. and Atreya, K. 2015. Sustainable livelihoods through conservation of wetland resources: a case of economic benefits from Ghodaghodi Lake, western Nepal. Ecology and Society 20(1):10. http://dx.doi.org/10.5751/ES07172-200110

Lamsal, P., Pant, K. P., Kumar, L. and Atreya, K. 2014. Diversity, uses, and threats in the Ghodaghodi Lake Complex, a Ramsar site in western lowland Nepal. ISRN Biodiversity 2014:1-12.

Li, A., Lei, G., Cao, X., Zhao, W., Deng, W. and Koirala, H. L. 2017. Land cover change and its driving forces in Nepal since 1990. In: Li A., Deng W., Zhao W. (Edss) Land cover change and its eco-environmental responses in Nepal. Springer Geography. Springer, Singapore. https://doi.org/10.1007/978-981-10-28908_3

Malla, R. 2009. Habitat mapping and conservation threats to river dolphin in Karnali river of Nepal. Banko Janakari 19(3):24-29. https://doi.org/10.3126/banko.v19i3.2208

Manel, S., Buckton, S. T. and Ormerod, S. J. 2000. Testing large-scale hypotheses using surveys: the effects of land use on the habitats, invertebrates and birds of Himalayan rivers. Journal of Applied Ecology 37:756-770. https://doi.org/10.1046/j.13652664.2000.00537.x 
Marzin, A., Archaimbault, V., Belliard, J., Chauvin, C., Delmas, F. and Pont, D. 2012. Ecological assessment of running waters: do macrophytes, macroinvertebrates, diatoms and fish show similar responses to human pressures? Ecological Indicators 23:56-65. https://doi.org/10.1016/j.ecolind.2012.03.010

Matangulu, M., Gurung, S., Prajapati, M. and Jyakhwo, R. 2017. Macroinvetebrate assemblages as indicators of water quality of the West Seti River, Bajhang, Nepal. International Journal of Environment 6:25-45. https://doi.org/10.3126/ije.v6i3.18096

Mitchell, J. and Zug, G. 1995. Keys to the known amphibians and reptiles of the Royal Chitwan National Park, Nepal. Smithsonian Herpetological Information Service 106:1-15.

Mitsch, W. J., Bernal, B. and Hernandez, M. E. 2015. Ecosystem services of wetlands. Taylor \& Francis. https://doi.org/10.1080/21513732.2015.1006250

MoFE. 2018. National Ramsar Strategy and Action Plan, Nepal (20182024). Ministry of Forests and Environment Singha Durbar, Kathmandu, Nepal.

Mohneke, M., Onadeko, A. B. and Rödel, M. O. 2011. Medicinal and dietary uses of amphibians in Burkina Faso. African Journal of Herpetology 60:78-83. https://doi.org/10.1080/21564574.2011.564660

Naiman, R. J. and Turner, M. G. 2000. A future perspective on North America's freshwater ecosystems. Ecological Applications 10:958-970.

Nanhoe, L. M. and Ouboter, P. E. 1987. The distribution of reptiles and amphibians in the Annapurna-Dhaulagiri region (Nepal). Rijksmuseum van Natuurlijke Historie, Leiden, The Netherlands.

Ng, H. H. and Edds, D. R. 2005. Two new species of Pseudecheneis, rheophilic catfishes (Teleostei: Sisoridae) from Nepal. Zootaxa 1047:1-19. https://doi.org/10.11646/zootaxa.1047.1.1

NLCDC. 2019. National Lake Conservation Development Committee. Kathmandu, Nepal. 2019, Government of Nepal.

NLCDC. 2020. Lake database of Nepal. National Lake Conservation Development Committee.

NPC. 2020. National wise distribution of hydro-projects. Government of Nepal, National Planning Commission.

https://www.npc.gov.np/nationwide_distribution_of_hydro_projects/

Olalde, I., Mallick, S., Patterson, N., Rohland, N., Villalba-Mouco, V., Silva, M. et al. 2019. The genomic history of the Iberian Peninsula over the past 8000 years. Science 363:1230-1234. https://doi.org/10.1126/science.aav4040

Ormerod, S. J., Rundle, S. D., Wilkinson, S. M., Daly, G. P., Dale, K. M. and Juttner, I. 1994. Altitudinal trends in the diatoms, bryophytes, macroinvertebrates and fish of a Nepalese river system. Freshwater Biology 32:309-322.

https://doi.org/10.1111/j.1365-2427.1994.tb01128.x

Paudel, S., Pal, P., Cove, M. V., Jnawali, S. R., Abel, G., Koprowski, J. L. et al. 2015a. The Endangered Ganges River dolphin Platanista gangetica gangetica in Nepal: abundance, habitat and conservation threats. Endangered Species Research 29:59-68. https://doi.org/10.3354/esr00702

Paudel, S., Timilsina, Y. P., Lewis, J., Ingersoll, T. and Jnawali, S. R. 2015b. Population status and habitat occupancy of endangered river dolphins in the Karnali River system of Nepal during low water season. Marine Mammal Science 31:707-719. https://doi.org/10.1111/mms.12192
Poudel, A. K. and Adhikari, D. 2020. Monitoring of Wetland Dependent Birds in Ramaroshan Lake Complex of Achham, Nepal. Bird Conservation Nepal, News Letter:12-14.

Poudel, K. and Sharma, S. 2012. Small irrigation systems in Nepal: An analytical preview from irrigated agricultural development perspective of batch I irrigation sub-projects under community managed irrigated agriculture sector project. Directorate of Agriculture Extension (DAE), Community Managed Irrigated Agriculture Sector Project (CMIASP), Harihar Bhawan, Lalitpur, Nepal.

Poudyal, L. P. and Chaudhary, H. 2019. Birds of Sukla Phanta National Park. 1st edition. Shuklaphanta National Park Office and Nepalese Ornithological Union, Kanchanpur and Kathmandu, Nepal.

Power, A. G. 2010. Ecosystem services and agriculture: tradeoffs and synergies. Philosophical Transactions of the Royal Society B: Biological Sciences 365:2959-2971.

https://doi.org/10.1098/rstb.2010.0143

Pradhan, N. M., Wegge, P., Moe, S. R. and Shrestha, A. K. 2008. Feeding ecology of two endangered sympatric megaherbivores: Asian elephant Elephas maximus and greater one-horned rhinoceros Rhinoceros unicornis in lowland Nepal. Wildlife Biology 14:147-154. https://doi.org/10.2981/0909-6396(2008)14[147:FEOTES]2.0.CO;2

Rodrigues, A. S., Andelman, S. J., Bakarr, M. I., Boitani, L., Brooks, T. M., Cowling, R. M. et al. 2004. Effectiveness of the global protected area network in representing species diversity. Nature 428:640. https://doi.org/10.1038/nature02422

Sah, J., Singh, R. and Bhatta, N. 2002. Floristic diversity and use of plants in Ghodaghodi lake area, Nepal. Journal of Natural History Museum 21:243-266.

Schleich, H. H. and Kästle, W. 2002. Amphibians and reptiles of Nepal: Biology, Systematics, Field Guide. ARG. Ganther Verlag KG, Ruggell, Germany.

Shah, D. N., Poudyal, A., Sharma, G., Levine, S., Subedi, N. and Dhakal, M. 2020a. Status, distribution, threats, and conservation of the Ganges River Dolphin Platanista gangetica (Mammalia: Artiodactyla: Cetacea) in Nepal. Journal of Threatened Taxa 12:15106-15113. https://doi.org/10.11609/jott.4397.12.1.1510615113

Shah, K. B. and Tiwari, S. 2004. Herpetofauna of Nepal: A Conservation Companion. IUCN Nepal, Kathmandu, Nepal.

Shah, R. D. T., Sharma, S., Shah, D. N. and Deepak, R. $2020 \mathrm{~b}$. Structure of benthic macroinvertebrate communities in the rivers of Western Himalaya, Nepal. Geosciences 10:150. https://doi.org/10.3390/geosciences10040150

Sharma, B., Rasul, G. and Chettri, N. 2015. The economic value of wetland ecosystem services: evidence from the Koshi Tappu Wildlife Reserve, Nepal. Ecosystem Services 12:84-93. https://doi.org/10.1016/j.ecoser.2015.02.007

Sharma, C. M. 2008. Freshwater fishes, fisheries, and habitat prospects of Nepal. Aquatic Ecosystem Health \& Management 11:289-297. https://doi.org/10.1080/14634980802317329

Shrestha, J. 1999. Coldwater fish and fisheries in Nepal. Pages 1340 in Petr, T., editor. Fish and fisheries at higher altitudes. Asia. FAO - Food and Agriculture Organization of the United Nations Rome, Italy. 
Shrestha, O. H. and Edds, D. R. 2012. Fishes of Nepal: Mapping distributions based on voucher specimens. Emporia State Research Studies 48:52-74.

Shrestha, T. 1990. Rare fishes of Himalayan waters of Nepal. Journal of Fish Biology 37:213-216.

Shrestha, T. K. 1989. Biology, status and conservation of the Ganges river dolphin, Platanista gangetica in Nepal. Pages 70-76 in W.F. Perrin, R.L. Borwell, Jr., Z. K., and Kang, L. J. (Eds) Biology and conservation of the river dolphins. IUCN Species Survival Commission.

Smith, B. D. 1994. Status, ecology and conservation of the Ganges river dolphin, Platanista gangetica, in the Karnali River, Nepal. Humboldt State University, USA.

Smith, M. and Battersby, J. 1953. LXVIII.-On a collection of amphibians and reptiles from Nepal. Annals and Magazine of Natural History 6:702-704. https://doi.org/10.1080/00222935308654472

Suren, A. M. 1994. Macroinvertebrate communities of streams in western Nepal: effects of altitude and land use. Freshwater Biology 32:323-336.

Terashima, A. 1984. Three new species of the cyprinid genus Schizothorax from Lake Rara, Northwestern Nepal. Japanese Journal of Ichthyology 31:122-135.

The World Bank. 2019. Nepal: modernizing irrigation system for economic growth and poverty reduction. The World Bank.

Timilsina, N., Tamang, B. and Baral, N. 2003. Status and conservation of Gangetic Dolphin (Platanista gangetica) in the Karnali River, Nepal. Tiger Paper 30:8-10.

Vörösmarty, C. J., Mclntyre, P. B., Gessner, M. O., Dudgeon, D., Prusevich, A., Green, P. et al. 2010. Global threats to human water security and river biodiversity. Nature 467:555. https://doi.org/10.1038/nature09440

Warkentin, I. G., Bickford, D., Sodhi, N. S. and Bradshaw, C. J. 2009. Eating frogs to extinction. Conservation Biology 23:1056-1059. https://www.jstor.org/stable/29738845

Webb, J. K., Brook, B. W. and Shine, R. 2002. Collectors endanger Australia's most threatened snake, the broad-headed snake Hoplocephalus bungaroides. Oryx 36:170-181.

WECS. 2003. Water resource strategy Nepal. Water and Energy Commission Secretaria (WECS), Kathmandu, Nepal.

WECS 2011. Water resources of Nepal in the context of climate change, Government of Nepal Water and Energy Commission Secretariat, Singha Durbar, Kathmandu

WWF. 2006. Status, distribution and conservation threats of Ganges River Dolphin in Karnali River, Nepal. WWF Nepal.

Zug, G. and Mitchell, J. 1995. Amphibians and reptiles of the Royal Chitwan National Park, Nepal. Asiatic Herpetological Research 6:172-18 